\title{
Reducibility for a Class of Two-Dimensional almost Periodic System with Small Perturbation
}

\author{
Fanhui Meng, Wenhua Qiu \\ School of Mathematics and Statistics, Zaozhuang University, Zaozhuang, China \\ Email: qiuwenhua2010@163.com
}

How to cite this paper: Meng, F.H. and Qiu, W.H. (2021) Reducibility for a Class of Two-Dimensional almost Periodic System with Small Perturbation. Advances in Pure Mathematics, 11, 950-962.

https://doi.org/10.4236/apm.2021.1112061

Received: November 13, 2021

Accepted: December 18, 2021

Published: December 21, 2021

Copyright $\odot 2021$ by author(s) and Scientific Research Publishing Inc. This work is licensed under the Creative Commons Attribution International License (CC BY 4.0). http://creativecommons.org/licenses/by/4.0/

\begin{abstract}
This paper focuses on the reducibility of two-dimensional almost periodic system with small perturbation. We use the KAM iterative method to get the reducibility by an almost periodic transformation. The system has been reduced to a simple form. So we have dealt with the small perturbation problem of the almost periodic system.
\end{abstract}

\section{Keywords}

Almost Periodic System, KAM Iterative Method, Reducibility

\section{Introduction}

The classical KAM theory which is developed in the last century by Kolmogorov, Arnold and Moser is the landmark of the development of Hamiltonian systems. The normal form theory is the earliest reducibility for linear ordinary differential equations by Poincaré. From then on, many people use KAM iterative method to study the reducibility of differential equations. In the last years, establishing the reducibility of finite-dimensional systems by the KAM tools is an active field of research, see [1] [2] [3] [4] [5] etc.

Junxiang $\mathrm{Xu}[6]$ considered the following quasi-periodic system:

$$
\left\{\begin{array}{l}
\dot{x}=\Omega y+h_{1}(x, y, t)+f_{1}(x, y, t) \\
\dot{y}=x^{3}+h_{2}(x, y, t)+f_{2}(x, y, t)
\end{array}\right.
$$

With the Diophantine condition and most of the sufficiently small parameters $\varepsilon$, the system can be changed to a suitable norm form by a real analytic quasi-periodic transformation. Similar, regular splitting and the general restricted linear equation are researched by the iterative method in [7] [8]. The existence 
of almost periodic solutions has also been widely attention, the small denominator condition is different from quasi-periodic system. However, relative to the rich results of quasi-periodic systems, the results of almost periodic systems are less, see [9] [10] etc.

In this paper, we consider the following almost periodic differential equation

$$
\left\{\begin{array}{l}
\dot{x}=\Omega y+h_{1}+f_{1} \\
\dot{y}=M(x, y)+h_{2}+f_{2}
\end{array}\right.
$$

where

$$
\begin{aligned}
M(x, y) & =m_{30} x^{3}+m_{20} x^{2}+m_{10} x+\left(m_{21} x^{2}+m_{11} x+m_{01}\right) y \\
& =\sum_{\mu=1}^{3} m_{\mu 0} x^{\mu}+\sum_{\mu=0}^{2} m_{\mu 1} x^{\mu} y .
\end{aligned}
$$

Our purpose is the reducibility of (2) with the almost periodic transformation. If $\|f\|_{m, \Delta_{r, s}} \leq \varepsilon$ with $f=\left(f_{1}, f_{2}\right)^{\mathrm{T}}$, there exists a real analytic almost-periodic transformation $\Phi$ :

$$
\left\{\begin{array}{l}
x=x_{+}+\xi\left(x_{+}, y_{+}, t\right), \\
y=y_{+}+\eta\left(x_{+}, y_{+}, t\right)
\end{array}\right.
$$

The system (2) can be changed to

$$
\left\{\begin{array}{l}
\dot{x}=\Omega y+h_{1}^{*}(x, y, t) \\
\dot{y}=M^{*}(x, y)+h_{2}^{*}(x, y, t) .
\end{array}\right.
$$

where $M^{*}(x, y)$ and $h^{*}(x, y, t)$ have the same form as $M$ and $h$.

\section{Some Definitions and Main Result}

We introduce some useful definitions.

Definition 2.1. The function $f(t)$ is called a quasi-periodic function of $t$ with frequencies $\omega_{1}, \omega_{2}, \cdots, \omega_{m}$, if there is a function $F(\theta)=F\left(\theta_{1}, \theta_{2}, \cdots, \theta_{m}\right)$, which is $2 \pi$-periodic in all its arguments $\theta_{i}(i=1, \cdots, m)$, such that $f(t)=F(\omega t)=F\left(\omega_{1} t, \omega_{2} t, \cdots, \omega_{m} t\right)$.

Write $D(0, r)=\left\{(x, y) \in C^{2}|| x|\leq r| y \mid, \leq r\right\}$, $T_{s}=\left\{\theta \in C^{m} / 2 \pi Z^{m}|| \operatorname{Im} \theta_{j} \mid \leq s, j=1,2, \cdots, m\right\}$, and $\Delta_{r, s}=D(0, r) \times T_{s}$.

Let $f(x, y, t)$ be real analytic in $x, y$ and $t$ on $\Delta_{r, s}$, and $f(x, y, t)$ be quasi-periodic with respect to $t$ with the frequency $\omega$. Then $f$ can be expanded as a Fourier series as follows.

$$
f(x, y, t)=\sum_{k \in Z^{m}} f_{k}(x, y) \mathrm{e}^{i\langle k, \theta\rangle} .
$$

We define the norm

$$
\|f\|_{\Delta_{r, s}}=\sum_{k \in Z^{m}}\left|f_{k}\right|_{r} \mathrm{e}^{|k| s},
$$

where $f_{k}(x, y)=\sum_{l, m} f_{l m k} x^{l} y^{m}$, and $\left|f_{k}\right|_{r}=\sup _{(x, y) \in D(0, r)} \sum_{l, m}\left|f_{l m k}\right||x|^{l}|y|^{m}$.

Definition 2.2. $N$ is the natural number set, $\tau$ is a set which is composed by 
the subset of $N$.

1) $\varnothing \in \tau$, if $\Lambda_{1}, \Lambda_{2} \in \tau$, then $\Lambda_{1} \cup \Lambda_{2} \in \tau$;

2) $\bigcup_{\Lambda \in \tau} \Lambda=N,[\varnothing]=0$ and $\left[\Lambda_{1} \cup \Lambda_{2}\right] \leq\left[\Lambda_{1}\right]+\left[\Lambda_{2}\right] \quad([\cdot]$ will be defined by next definition).

If $\tau$ satisfies the conditions above, we say that $(\tau,[\cdot])$ is the finite spatial structure on $N$.

Definition 2.3. A function $\Delta$ is called an approximation function, if it satisfies:

1) $\Delta:[0,+\infty) \rightarrow[1,+\infty), \Delta(0)=1$, and $\Delta$ is a nondecreasing function,

2) $\log \Delta(t) / t$ is a decreasing function in $[0,+\infty)$,

3) $\int_{0}^{\infty} \log \Delta(t) / t^{2}<+\infty$.

Definition 2.4. Let $f(x, y, t)=\sum_{\Lambda \in \tau} f_{\Lambda}(x, y, t)$ with the frequency

$\omega=\left(\omega_{1}, \omega_{2}, \cdots\right)$. We define the weight norm of $f(x, y, t)$ in the finite spatial structure $(\tau,[\cdot])$ as follows.

For $m \geq 0, s \geq 0$,

$$
\|f(x, y, t)\|_{m, \Delta_{r, s}}=\sum_{\Lambda \in \tau} \mathrm{e}^{m[\Lambda]}\left\|f_{\Lambda}(x, y, t)\right\|_{\Delta_{r, s}} .
$$

Let

$$
\mathcal{A}_{r, s}^{m}=\left\{f \mid\|f\|_{m, \Delta_{r, s}}<\infty\right\} .
$$

Now we state the main result of this paper.

Theorem 2.5. We consider system $(2), h=\left(h_{1}, h_{2}\right)^{\mathrm{T}}$ and $f=\left(f_{1}, f_{2}\right)^{\mathrm{T}}$ are analytic almost-periodic in $t$ with the frequency vector $\omega=\left(\omega_{1}, \omega_{2}, \cdots\right)$, h are higher order terms with

$$
h(x, y, t)=\sum_{v \geq 2 \text { or } \mu+v \geq 4} h_{\mu v}(t) x^{\mu} y^{v},
$$

and $f$ are lower order terms with

$$
f(x, y, t)=\sum_{v \leq 1, \mu+v \leq 3} f_{\mu \nu}(t) x^{\mu} y^{v} .
$$

Suppose

$$
|\langle k, \omega\rangle| \geq \frac{\alpha}{\Delta^{4}(|k|) \Delta^{4}([k])}, k \in Z^{\infty} \backslash\{0\},
$$

where $\alpha>0$ is a constant and $\Delta$ is an approximation function.

Then there exists $\varepsilon>0$, such that if $\|f\|_{m, \Delta_{r, s}} \leq \varepsilon$, there exists a real analytic almost-periodic transformation $\Phi$ :

$$
\begin{gathered}
\left\{\begin{array}{l}
x=x_{+}+\xi\left(x_{+}, y_{+}, t\right), \\
y=y_{+}+\eta\left(x_{+}, y_{+}, t\right)
\end{array}\right. \\
\Phi(\cdot,, t):\left(x_{+}, y_{+}\right) \in D\left(0, \frac{r}{2}\right) \rightarrow(x, y) \in D(0, r), \text { so system }(2) \text { is changed to } \\
\left\{\begin{array}{l}
\dot{x}=\Omega y+h_{1}^{*}(x, y, t) \\
\dot{y}=M^{*}(x, y)+h_{2}^{*}(x, y, t)
\end{array}\right.
\end{gathered}
$$


where $M^{*}(x, y)=m_{30}^{*} x^{3}+m_{20}^{*} x^{2}+m_{10}^{*} x+\left(m_{21}^{*} x^{2}+m_{11}^{*} x+m_{01}^{*}\right) y$, and $h^{*}(x, y, t)=\left(h_{1}^{*}(x, y, t), h_{2}^{*}(x, y, t)\right)^{\mathrm{T}}$ has the same form as $h$.

\section{Solving Homological Equations}

We will use the modified KAM iteration to proof Theorem 2.5. In this section, We will solve the homological equations, which is in any step.

Let

$$
\begin{aligned}
& \xi(x, y, t)=\xi_{0}(x, t)+\xi_{1}(x, t) y, \\
& \eta(x, y, t)=\eta_{0}(x, t)+\eta_{1}(x, t) y,
\end{aligned}
$$

where

$$
\xi_{0}(x, y, t)=\sum_{\mu \leq 3} \xi_{\mu 0}(t) x^{\mu}, \xi_{1}(x, y, t)=\sum_{\mu \leq 2} \xi_{\mu 1}(t) x^{\mu},
$$

and $\eta_{0}, \eta_{1}$ have the same form as $\xi_{0}, \xi_{1}$.

Let $D=\frac{\partial(\xi, \eta)}{\partial(x, y)}$ be the Jacobian matrix. Define the almost-periodic transformation $\Phi^{\prime}(\cdot, \cdot, t):\left(x_{+}, y_{+}\right) \rightarrow(x, y)$ by

$$
\left\{\begin{array}{l}
x=x_{+}+\xi\left(x_{+}, y_{+}, t\right), \\
y=y_{+}+\eta\left(x_{+}, y_{+}, t\right)
\end{array}\right.
$$

Then the system is transformed into

$$
\left\{\begin{array}{l}
\dot{x}_{+}=\Omega y_{+}+G_{1}\left(x_{+}, y_{+}, t\right)+f_{1}\left(x_{+}, y_{+}, t\right)+\tilde{P}_{1}\left(x_{+}, y_{+}, t\right) \\
\dot{y}_{+}=M\left(x_{+}, y_{+}, t\right)+G_{2}\left(x_{+}, y_{+}, t\right)+f_{2}\left(x_{+}, y_{+}, t\right)+\tilde{P}_{2}\left(x_{+}, y_{+}, t\right),
\end{array}\right.
$$

and

$$
\left\{\begin{array}{l}
G_{1}=\left(\Omega \eta_{0}-\partial_{t} \xi_{0}\right)+\left(\Omega \eta_{1}-\partial_{t} \xi_{1}\right) y_{+}, \\
G_{2}=\left(M_{1} \eta_{0}+\frac{\partial M_{0}}{\partial x_{+}} \xi_{0}-\partial_{t} \eta_{0}\right)+\left(M_{1} \eta_{1}+\frac{\partial M_{0}}{\partial x_{+}} \xi_{1}+\frac{\partial M_{1}}{\partial x_{+}} \xi_{0}-\partial_{t} \eta_{1}\right) y_{+},
\end{array}\right.
$$

where

$$
\tilde{P}=\left(\tilde{P}_{1}, \tilde{P}_{2}\right)^{\mathrm{T}}=(I+D)^{-1}\left[-D L-D f-D F_{1}+D F_{2}+F_{3}+\left(f \circ \Phi^{\prime}-f\right)+h \circ \Phi^{\prime}\right],
$$

and

$$
\begin{gathered}
L=\left[\begin{array}{c}
\Omega y_{+} \\
M\left(x_{+}, y_{+}\right)
\end{array}\right], \\
F_{1}=\left[\begin{array}{c}
\Omega \eta \\
\frac{\mathrm{d} M_{0}}{\mathrm{~d} x_{+}} \xi+\frac{\mathrm{d} M_{1}}{\mathrm{~d} x_{+}} \xi \eta+M_{1}\left(x_{+}, t\right) \eta
\end{array}\right], \\
F_{2}=\left[\begin{array}{c}
\partial_{t} \xi \\
\partial_{t} \eta
\end{array}\right], \\
F_{3}=\left[\begin{array}{c}
0 \\
F
\end{array}\right]
\end{gathered}
$$


with

$$
F=\frac{1}{2} \frac{\partial^{2} M_{0}}{\partial x_{+}^{2}}\left(x_{+}^{\prime}, t\right) \xi^{2}+\frac{1}{2} \frac{\partial^{2} M_{1}}{\partial x_{+}^{2}}\left(x_{+}^{\prime}, t\right) \xi^{2}+\frac{\partial M_{1}}{\partial x_{+}}\left(x_{+}^{\prime}, t\right) \xi \eta+\frac{\partial M_{1}}{\partial x_{+}}\left(x_{+}^{\prime}, t\right) \xi_{1} y^{2},
$$

here $x_{+}^{\prime}$ between $x$ and $x_{+}, F$ are the remainders of Taylor.

Our aim is that $\left(\Omega y_{+}+G_{1}+f_{1}, M+G_{2}+f_{2}\right)^{\mathrm{T}}$ will be a new normal form and $\left(\tilde{P}_{1}, \tilde{P}_{2}\right)^{\mathrm{T}}$ will be much smaller perturbation. So, the system will converge to a suitable normal form by iterated infinitely. Note that below we use $(x, y)$ in place of $\left(x_{+}, y_{+}\right)$to simplify the formulas.

Let $f_{i}(x, y, t)=f_{i 0}(x, t)+f_{i 1}(x, t) y$, where

$$
f_{i 0}(x, t)=\sum_{\mu=0}^{3} f_{i, \mu 0}(t) x^{\mu}
$$

and

$$
f_{i 1}(x, t)=\sum_{\mu=0}^{2} f_{i, \mu 1}(t) x^{\mu} .
$$

From (4) and (5), we will solve the following homological equations, we hope to find $\xi_{0}, \xi_{1}, \eta_{0}, \eta_{1}$ and $\hat{M}_{0}, \hat{M}_{1}$.

$$
\left\{\begin{array}{l}
\Omega \eta_{0}-\partial_{t} \xi_{0}=-f_{1,0}, \\
\Omega \eta_{1}-\partial_{t} \xi_{1}=-f_{1,1}, \\
M_{1} \eta_{0}+\frac{\partial M_{0}}{\partial x} \xi_{0}-\partial_{t} \eta_{0}=-f_{2,0}+g_{0}+\hat{M}_{0}, \\
M_{1} \eta_{1}+\frac{\partial M_{0}}{\partial x} \xi_{1}+\frac{\partial M_{1}}{\partial x} \xi_{0}-\partial_{t} \eta_{1}=-f_{2,1}+g_{1}+\hat{M}_{1}
\end{array}\right.
$$

and

$$
\begin{aligned}
g_{0}(x, y, t)= & \sum_{\mu^{\prime}+\mu^{\prime \prime}=\mu, j+j^{\prime}=\mu, \mu \geq 3}\left[m_{\mu^{\prime} 1} \eta_{\mu^{\prime \prime} 0}+\left(j^{\prime}+1\right) \xi_{j 0} m_{\left(j^{\prime}+1\right) 0}\right] x^{\mu}, \\
g_{1}(x, y, t)= & \sum_{l+l^{\prime}=\mu, n+n^{\prime}=\mu, \mu^{\prime}+\mu^{\prime \prime}=\mu, \mu \geq 2}\left[m_{l 1} \eta_{l^{\prime} 1}+(n+1) m_{(n+1) 0} \xi_{n^{\prime} 1}\right. \\
& \left.+\left(\mu^{\prime}+1\right) m_{\left(\mu^{\prime}+1\right) 1} \xi_{\mu^{\prime \prime} 0}\right] x^{\mu} .
\end{aligned}
$$

Then $g=g_{0}(x, t)+g_{1}(x, t) y$, and $g_{0}, g_{1}$ are high-order terms of $x, y$. They will be put into the new perturbation.

Now, we can compare the coefficients of $x^{\mu}$ in the equations, and we get

$$
\left\{\begin{array}{l}
\Omega \eta_{\mu 0}-\partial_{t} \xi_{\mu 0}=-f_{1, \mu 0}, \\
\Omega \eta_{\mu 1}-\partial_{t} \xi_{\mu 1}=-f_{1, \mu 1}, \\
\sum_{\mu^{\prime}+\mu^{\prime \prime}=\mu} m_{\mu^{\prime} 1} \eta_{\mu^{\prime \prime} 0}+\sum_{\mu^{\prime}+\mu^{\prime \prime}=\mu}\left(\mu^{\prime \prime}+1\right) \xi_{\mu^{\prime} 0} m_{\left(\mu^{\prime \prime}+1\right) 0}-\partial_{t} \eta_{\mu 0}=-f_{2, \mu 0}+\hat{m}_{\mu 0}, \\
\sum_{\mu^{\prime}+\mu^{\prime \prime}=\mu} \eta_{\mu^{\prime} 1} m_{\mu^{\prime \prime} 1}+\sum_{\mu^{\prime}+\mu^{\prime \prime}=\mu}\left(\mu^{\prime \prime}+1\right) \xi_{\mu^{\prime} 1} m_{\left(\mu^{\prime \prime}+1\right) 0} \\
+\sum_{\mu^{\prime}+\mu^{\prime \prime}=\mu}\left(\mu^{\prime \prime}+1\right) \xi_{\mu^{\prime} 0} m_{\left(\mu^{\prime \prime}+1\right) 1}-\partial_{t} \eta_{\mu 1}=-f_{2, \mu 1}+\hat{m}_{\mu 1} .
\end{array}\right.
$$

Note that $m_{00}=0$, we take the first and third equations of (7) with $\mu=0$, so we get 


$$
\left\{\begin{array}{l}
\Omega \eta_{00}-\partial_{t} \xi_{00}=-f_{1,00}, \\
m_{01} \eta_{00}+\xi_{00} m_{10}-\partial_{t} \eta_{00}=-f_{2,00}+\hat{m}_{00} .
\end{array}\right.
$$

Let

$$
f_{i, 00}=\sum_{\Lambda \in \tau} f_{i, 00 \Lambda}, \quad f_{i, 00 \Lambda}=\sum_{\text {suppk } \subset \Lambda} f_{i, 00 \Lambda k} \mathrm{e}^{i\langle k, \omega t\rangle}, i=1,2,
$$

and

$$
\begin{array}{ll}
\xi_{00}=\sum_{\Lambda \in \tau} \xi_{00 \Lambda}, & \xi_{00 \Lambda}=\sum_{\text {suppk } \subset \Lambda} \xi_{00 \Lambda k} \mathrm{e}^{i\langle k, \omega t\rangle}, \\
\eta_{00}=\sum_{\Lambda \in \tau} \eta_{00 \Lambda}, & \eta_{00 \Lambda}=\sum_{\text {suppk } \subset \Lambda} \eta_{00 \Lambda k} \mathrm{e}^{i\langle k, \omega t\rangle} .
\end{array}
$$

Then we have

$$
\left\{\begin{array}{l}
\Omega \eta_{00 \Lambda k}+\lambda \xi_{00 \Lambda k}=-f_{1,00 \Lambda k}, \\
m_{01} \eta_{00 \Lambda k}+\xi_{00 \Lambda k} m_{10}+\lambda \eta_{00 \Lambda k}=-f_{2,00 \Lambda k}+\hat{m}_{00}
\end{array}\right.
$$

with $\lambda=-i\langle k, \omega\rangle$.

If $k=0$, we get

$$
\left\{\begin{array}{l}
\eta_{00 \Lambda 0}=-f_{1,00 \Lambda 0} / \Omega, \\
\hat{m}_{00}=m_{01} \eta_{00 \Lambda 0}+\xi_{00 \Lambda 0} m_{10}+f_{2,00 \Lambda 0} .
\end{array}\right.
$$

Then the solution can be got.

If $k \neq 0$, we have $\mathrm{Hz}=d$ with

$$
z=\left(\xi_{00 \Lambda k}, \eta_{00 \Lambda k}\right)^{\mathrm{T}}, d=-\left(f_{1,00 \Lambda k}, f_{2,00 \Lambda k}\right)^{\mathrm{T}} .
$$

So

$$
H=\left[\begin{array}{cc}
\lambda & \Omega \\
m_{10} & m_{01}+\lambda
\end{array}\right]
$$

And

$$
\operatorname{det}(H)=\lambda^{2}+m_{01} \lambda-m_{10} \Omega=i\langle k, \omega\rangle m_{01}-\left(|\langle k, \omega\rangle|^{2}+m_{10} \Omega\right) .
$$

So we have

$$
|\operatorname{det}(H)| \geq|\langle k, \omega\rangle|^{2}+m_{10} \Omega \geq \frac{\alpha^{2}}{\Delta^{8}(|k|) \Delta^{8}([k])}
$$

with $m_{10} \geq 0, \Omega \geq 0$. Thus the Equations (9) are solved with $z=H^{-1} d$, and $z$ has the same finite spatial structure with $f$.

So

$$
\begin{aligned}
& \left\|\xi_{00 \Lambda}\right\|_{\delta-3 \rho} \leq\left\|\xi_{00 \Lambda}\right\|_{\delta-2 \rho} \leq \sum_{\text {suppkㅅ }} \frac{\Delta^{8}(|k|) \Delta^{8}([k])}{\alpha^{2}}\left|f_{i, 00 \Lambda k}\right| \mathrm{e}^{(\delta-2 \rho)|k|} \\
& =\sum_{\text {suppk } \subset \Lambda} \frac{\Delta^{8}(|k|) \mathrm{e}^{-2 \rho|k|}}{\alpha^{2}} \Delta^{8}([k])\left|f_{i, 00 \Lambda k}\right| \mathrm{e}^{\delta|k|} \\
& \leq \frac{\Gamma^{2}(\rho) \Delta^{8}([\Lambda])}{\alpha^{2}}\left\|f_{i, 00 \Lambda}\right\|_{\delta} \text {. }
\end{aligned}
$$


where $\Gamma(\rho)=\sup _{t \geq 0}\left(\Delta^{4}(t) \mathrm{e}^{-\rho t}\right)$.

It follows that

$$
\begin{aligned}
\left\|\xi_{00}\right\|_{m-3 \bar{m}, \delta-3 \rho} & \leq \sum_{\Lambda \in \tau}\left\|\xi_{00 \Lambda}\right\|_{\delta-2 \rho} \mathrm{e}^{(m-2 \bar{m})[\Lambda]} \\
& \leq \sum_{\Lambda \in \tau} \frac{\Gamma^{2}(\rho) \Delta^{8}([\Lambda]) \mathrm{e}^{-2 \bar{m}[\Lambda]}}{\alpha^{2}}\left\|f_{i, 00 \Lambda}\right\|_{\delta} \mathrm{e}^{m[\Lambda]} \\
& \leq \frac{\Gamma^{2}(\bar{m}) \Gamma^{2}(\rho)}{\alpha^{2}}\left\|f_{i, 00}\right\|_{m, \delta} \\
& \leq \frac{\Gamma^{2}(\bar{m}) \Gamma^{2}(\rho)}{\alpha^{2}} \varepsilon
\end{aligned}
$$

We can also get

$$
\left\|\eta_{00}\right\|_{m-3 \bar{m}, \delta-3 \rho} \leq \frac{\Gamma^{2}(\bar{m}) \Gamma^{2}(\rho)}{\alpha^{2}} \varepsilon .
$$

Now we replace $\mu$ with $\mu+1$ in the first, third equations of (7) and then obtain

$$
\left\{\begin{array}{l}
\Omega \eta_{(\mu+1) 0}-\partial_{t} \xi_{(\mu+1) 0}=d_{1}, \\
\Omega \eta_{\mu 1}-\partial_{t} \xi_{\mu 1}=d_{2}, \\
m_{01} \eta_{(\mu+1) 0}+\xi_{(\mu+1) 0} m_{10}-\partial_{t} \eta_{(\mu+1) 0}=d_{3}+\hat{m}_{(\mu+1) 0}, \\
\eta_{\mu 1} m_{01}+\xi_{\mu 1} m_{10}-\partial_{t} \eta_{\mu 1}=d_{4}+\hat{m}_{\mu 1} .
\end{array}\right.
$$

where $l=0,1,2$, and

$$
\left\{\begin{aligned}
d_{1}= & -f_{1,(\mu+1) 0}, \\
d_{2}= & -f_{1, \mu 1}, \\
d_{3}= & -f_{2,(\mu+1) 0}-m_{11} \eta_{(\mu) 0}-m_{21} \eta_{(\mu-1) 0}-\cdots-m_{(\mu+1) 1} \eta_{00} \\
& -2 \xi_{\mu 0} m_{20}-3 \xi_{(\mu-1) 0} m_{30}-\cdots-(\mu+2) \xi_{00} m_{(\mu+2) 0}, \\
d_{4}= & -f_{2, \mu 1}-\eta_{(\mu-1) 1} m_{11}-\eta_{(\mu-2) 1} m_{21}-\cdots-\eta_{01} m_{\mu 1} \\
& -2 \xi_{(\mu-1) 1} m_{20}-3 \xi_{(\mu-2) 1} m_{30}-\cdots-(\mu+1) \xi_{01} m_{(\mu+1) 0} \\
& -\sum_{\mu^{\prime}+\mu^{\prime \prime}=\mu}\left(\mu^{\prime \prime}+1\right) \xi_{\mu^{\prime} 0} m_{\left(\mu^{\prime \prime}+1\right) 1} .
\end{aligned}\right.
$$

Let $d=\left(d_{1}, d_{2}, d_{3}, d_{4}\right)^{\mathrm{T}}$,

$$
z=\left(\xi_{(\mu+1) 0}, \xi_{\mu 1}, \eta_{(\mu+1) 0}, \eta_{\mu 1}\right)^{\mathrm{T}} .
$$

Let

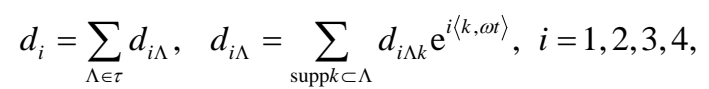

and

$$
\begin{aligned}
& \xi_{(\mu+1) 0}=\sum_{\Lambda \in \tau} \xi_{(\mu+1) 0 \Lambda}, \quad \xi_{(\mu+1) 0 \Lambda}=\sum_{\text {suppkㅅ }} \xi_{(\mu+1) 0 \Lambda k} \mathrm{e}^{i\langle k, \omega t\rangle}, \\
& \xi_{\mu 1}=\sum_{\Lambda \in \tau} \xi_{\mu 1 \Lambda}, \quad \xi_{\mu 1 \Lambda}=\sum_{\text {suppk } \subset \Lambda} \xi_{\mu 1 \Lambda k} \mathrm{e}^{i\langle k, \omega t\rangle},
\end{aligned}
$$

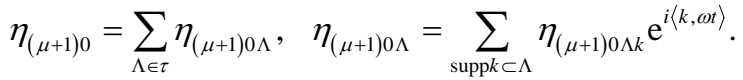




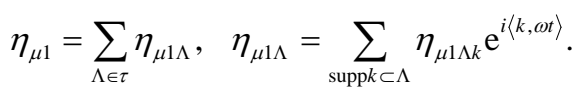

Then the coefficient matrix

$$
H=\left[\begin{array}{cccc}
\lambda & 0 & \Omega & 0 \\
0 & \lambda & 0 & \Omega \\
m_{10} & 0 & m_{01}+\lambda & 0 \\
0 & m_{10} & 0 & m_{01}+\lambda
\end{array}\right]
$$

with $\lambda=-i\langle k, \omega\rangle$.

$$
\operatorname{det}(H)=\lambda^{4}+\left(m_{01}^{2}-2 \Omega m_{10}\right) \lambda^{2}+\Omega^{2} m_{10}^{2} 2 m_{01} \lambda^{3}-2 \Omega m_{10} \lambda .
$$

The second part is the imaginary part of $\operatorname{det}(H)$, so

$$
|\operatorname{Im} \operatorname{det}(H)|=2 m_{01}|\langle k, \omega\rangle|^{3}+2 \Omega m_{01} m_{10}|\langle k, \omega\rangle| \geq \frac{\alpha^{3}}{\Delta^{12}(|k|) \Delta^{12}([k])}
$$

with $m_{01} \geq 0, m_{10} \geq 0$.

Thus,

$$
\begin{aligned}
\left\|\xi_{(\mu+1) 0 \Lambda}\right\|_{\delta-3 \rho} & \leq \sum_{\text {suppk } \subset \Lambda} \frac{\Delta^{12}(|k|) \Delta^{12}([k])}{\alpha^{3}}\left|d_{i \Lambda k}\right| \mathrm{e}^{(\delta-3 \rho)|k|} \\
& =\sum_{\text {suppk } \subset \Lambda} \frac{\Delta^{12}(|k|) \mathrm{e}^{-3 \rho|k|}}{\alpha^{3}} \Delta^{12}([k])\left|d_{i \Lambda k}\right| \mathrm{e}^{\delta|k|} \\
& \leq \frac{\Gamma^{3}(\rho) \Delta^{12}([\Lambda])}{\alpha^{3}}\left\|d_{i \Lambda}\right\|_{\delta} .
\end{aligned}
$$

It follows that

$$
\begin{aligned}
\left\|\xi_{(\mu+1) 0}\right\| \|_{m-3 \bar{m}, \delta-3 \rho} & =\sum_{\Lambda \in \tau}\left\|\xi_{(\mu+1) 0 \Lambda}\right\|_{\delta-3 \rho} \mathrm{e}^{(m-3 \bar{m})[\Lambda]} \\
& \leq \sum_{\Lambda \in \tau} \frac{\Gamma^{3}(\rho) \Delta^{12}([\Lambda]) \mathrm{e}^{-3 \bar{m}[\Lambda]}}{\alpha^{3}}\left\|d_{i \Lambda}\right\|_{\delta} \mathrm{e}^{m[\Lambda]} \\
& \leq \frac{\Gamma^{3}(\bar{m}) \Gamma^{3}(\rho)}{\alpha^{3}}\left\|d_{i}\right\|_{m, \delta} \\
& \leq \frac{\Gamma^{3}(\bar{m}) \Gamma^{3}(\rho)}{\alpha^{3}} \varepsilon,
\end{aligned}
$$

For $k=0, \mu=0,1,2$, we let

$$
\xi_{(\mu+1) 0 \Lambda 0}=0, \xi_{\mu 1 \Lambda 0}=0,
$$

and

$$
\begin{gathered}
\eta_{(\mu+1) 0 \Lambda 0}=-f_{1,(\mu+1) 0 \Lambda 0} / \Omega, \eta_{\mu 1 \Lambda 0}=-f_{1, \mu 1 \Lambda 0} / \Omega \\
\hat{m}_{(\mu+1) 0}=m_{01} \eta_{(\mu+1) 0 \Lambda 0}-d_{3 \Lambda 0}, \\
\hat{m}_{\mu 1}=m_{01} \eta_{\mu 1 \Lambda 0}-d_{4 \Lambda 0} .
\end{gathered}
$$

So (11) are solved with

$$
\|z\|_{m-3 \bar{m}, \delta-3 \rho} \leq \frac{\Gamma^{3}(\bar{m}) \Gamma^{3}(\rho)}{\alpha^{3}} \varepsilon .
$$


When we have solved (7) which the order of $x$ and $y$ is no more than $\mu$, the system can be solved inductively for $\mu+1$. So we can solve system (7) and obtain $\xi_{\mu v}, \eta_{\mu v}$ with

$$
\left\|\xi_{\mu v}\right\|\left\|_{m-3 \bar{m}, \delta-3 \rho} \leq c \frac{\Gamma^{3}(\bar{m}) \Gamma^{3}(\rho)}{\alpha^{3}} \varepsilon,\right\| \eta_{\mu v} \|_{m-3 \bar{m}, \delta-3 \rho} \leq c \frac{\Gamma^{3}(\bar{m}) \Gamma^{3}(\rho)}{\alpha^{3}} \varepsilon,
$$

Noting that $\mu+v \leq 3$, we have

$$
\|\xi\|_{m-3 \bar{m}, \Delta(r, \delta-3 \rho)} \leq c \frac{\Gamma^{3}(\bar{m}) \Gamma^{3}(\rho)}{\alpha^{3}} \varepsilon,\|\eta\|_{m-3 \bar{m}, \Delta(r, \delta-3 \rho)} \leq c \frac{\Gamma^{3}(\bar{m}) \Gamma^{3}(\rho)}{\alpha^{3}} \varepsilon .
$$

Furthermore, by (14) it follows that

$$
\|D\| \|_{m-3 \bar{m}, \Delta(r, \delta-3 \rho)} \leq c \frac{\Gamma^{3}(\bar{m}) \Gamma^{3}(\rho)}{\alpha^{3}} \varepsilon .
$$

Now we consider $\hat{M}(x, y)=\hat{M}_{0}(x)+\hat{M}_{1}(x) y$ with

$$
\hat{M}_{0}(x)=\sum_{1 \leq \mu \leq 3} \hat{m}_{\mu 0} x^{\mu}, \hat{M}_{1}(x)=\sum_{\mu \leq 2} \hat{m}_{\mu 1} x^{\mu} .
$$

Let

$$
\begin{gathered}
\tilde{M}(x, y)=M(x, y)+\hat{M}(x, y)=\tilde{M}_{0}(x)+\tilde{M}_{1}(x) y_{2}, \\
\tilde{M}_{0}(x)=M_{0}(x)+\hat{M}_{0}(x)=\sum_{1 \leq \mu \leq 3} m_{\mu 0} x^{\mu}+\sum_{1 \leq \mu \leq 3} \hat{m}_{\mu 0} x^{\mu}, \\
\tilde{M}_{1}(x)=M_{1}(x)+\hat{M}_{1}(x)=\sum_{\mu \leq 2} m_{\mu 1} x^{\mu}+\sum_{\mu \leq 2} \hat{m}_{\mu 1} x^{\mu} .
\end{gathered}
$$

Now we want to change $\tilde{M}(x, y)$ to normal form with zero as an equilibrium point. Actually, this problem is the stability of $M_{0}(x)$ under small perturbations. From Theorem 2.2 in [8], we know that $\tilde{M}_{0}(x)$ has a real root $x_{(0)}$, such that $\left|X_{(0)}\right| \leq c \varepsilon$. In the KAM iterations, $M_{0}(x)$ and $\hat{M}_{0}(x)$ correspond to $f_{j}$ and $\hat{f}_{j}$ in Theorem $2.2[8]$.

Let $\Phi^{\prime \prime}: x=x_{+}+x_{(0)}, y=y_{+}$then we have

$$
M^{+}(x, y)=\tilde{M} \circ \Phi^{\prime \prime}(x, y)=\tilde{M}\left(x+x_{(0)}, y\right)=M_{0}^{+}+M_{1}^{+} y
$$

with $M_{0}^{+}=\sum_{1 \leq \mu \leq 3} m_{\mu 0}^{+} x^{\mu}$. Moreover we have

$$
\left\|M^{+}-M\right\|_{r} \leq c \varepsilon
$$

Let

$$
\begin{gathered}
\Phi(x, y, t)=\Phi^{\prime} \circ \Phi^{\prime \prime}(x, y, t), \\
\left\{\begin{array}{l}
x=x_{+}+x_{(0)}+\xi\left(x_{+}+x_{(0)}, y_{+}, t\right), \\
y=y_{+}+\eta\left(x_{+}+x_{(0)}, y_{+}, t\right) .
\end{array}\right.
\end{gathered}
$$

Then system (2) can be transformed into

$$
\left\{\begin{array}{l}
\dot{x}=\Omega y+P_{1}^{+}(x, y, t), \\
\dot{y}=M^{+}(x, y)+P_{2}^{+}(x, y, t),
\end{array}\right.
$$


where $P^{+}=\tilde{P} \circ \Phi^{\prime \prime}+g \circ \Phi^{\prime \prime}$. By the express of $\tilde{P}$, we get

$$
\begin{aligned}
P^{+}= & (I+\tilde{D})^{-1}\left[-\tilde{D} L \circ \Phi^{\prime \prime}-\tilde{D} f \circ \Phi^{\prime \prime}-\tilde{D} F_{1} \circ \Phi^{\prime \prime}+\tilde{D} F_{2} \circ \Phi^{\prime \prime}+F_{3} \circ \Phi^{\prime \prime}\right. \\
& \left.+\left(f \circ \Phi^{\prime} \circ \Phi^{\prime \prime}-f \circ \Phi^{\prime \prime}\right)+h \circ \Phi^{\prime} \circ \Phi^{\prime \prime}\right]+g \circ \Phi^{\prime \prime},
\end{aligned}
$$

with $\tilde{D}=D \circ \Phi^{\prime \prime}$.

Let

$$
\varepsilon_{+}=\frac{\Gamma^{3}(\bar{m}) \Gamma^{3}(\rho)}{\alpha^{3}} \varepsilon, r_{+}=r-4 \hat{r}, m_{+}=m-3 \bar{m}, \delta_{+}=\delta-3 \rho .
$$

$h^{+}=P_{H}^{+}$are higher order terms of $P^{+}$,

$$
h^{+}=\sum_{v \leq 1 \text { and } \mu+v \geq 4} h_{\mu \nu}^{+}(t) x^{\mu} y^{v}+\sum_{v \geq 2} h_{\mu \nu}^{+}(t) x^{\mu} y^{v} .
$$

$f^{+}=P_{L}^{+}$are lower order terms of $P^{+}$,

$$
f^{+}(x, y, t)=\sum_{v \leq 1, \mu+v \leq 3} f_{\mu \nu}^{+}(t) x^{\mu} y^{v} .
$$

From above estimation of the transformation and the similar method in [8], we can have

$$
\begin{gathered}
\left\|f^{+}\right\| \|_{m_{+}, \Delta\left(r_{+}, \delta_{+}\right)} \leq \varepsilon_{+} . \\
\left\|h^{+}-h\right\|_{m_{+}, \Delta\left(r_{+}, \delta_{+}\right)} \leq \varepsilon_{+} .
\end{gathered}
$$

Now the system of differential equations becomes

$$
\left\{\begin{array}{l}
\dot{x}=\Omega y+h_{1}^{+}(x, y, t)+f_{1}^{+}(x, y, t), \\
\dot{y}=M^{+}(x, y)+h_{2}^{+}(x, y, t)+f_{2}^{+}(x, y, t) .
\end{array}\right.
$$

\section{Proof of Theorem 2.5}

Now we consider system (2):

$$
\left\{\begin{array}{l}
\dot{x}=\Omega y+h_{1}+f_{1} \\
\dot{y}=M(x, y)+h_{2}+f_{2}
\end{array}\right.
$$

We will transform this system to (3) by iterative infinitely. First of all, we choose the following parameters as initial values:

$$
r_{0}=r, \hat{r}_{0}=r / 16, \delta_{0}=\delta, \rho_{0}=\delta / 10,
$$

and

$$
m_{0}=\delta / 4, \bar{m}_{0}=\delta / 16, \varepsilon_{0}=\varepsilon .
$$

The $j$-th step as follows:

$$
\begin{gathered}
r_{j}=r_{j-1}-4 \hat{r}_{j-1}, \hat{r}_{j}=\hat{r}_{j-1} / 2, \delta_{j}=\delta_{j-1}-3 \rho_{j-1}, \rho_{j}=\rho_{j-1} / 2, \\
m_{j}=m_{j-1}-3 \bar{m}_{j-1}, \bar{m}_{j}=\bar{m}_{j-1} / 2, \varepsilon_{j+1}=\frac{\Gamma^{3}\left(\bar{m}_{j}\right) \Gamma^{3}\left(\rho_{j}\right)}{\alpha^{3}} \varepsilon_{j} .
\end{gathered}
$$

From Section 3, we can get a sequence of almost-periodic transformations $\Phi_{j}(\cdot,, t)$ with $t$ on $T_{\delta_{j}}, j=1,2, \cdots$, such that 


$$
\begin{gathered}
\Phi_{j}(\cdot, \cdot, t): D\left(0, r_{j}\right) \rightarrow D\left(0, r_{j-1}-\hat{r}_{j-1}\right) \subset D\left(0, r_{j-1}\right) . \\
\left\{\begin{array}{l}
x_{j-1}=x_{j}+x_{(j)}+\xi_{j}\left(x_{j}+x_{(j)}, y_{j}, t\right), \\
y_{j-1}=y_{j}+\eta_{j}\left(x_{j}+x_{(j)}, y_{j}, t\right)
\end{array}\right.
\end{gathered}
$$

Let $\Phi^{j}=\Phi_{1} \circ \cdots \Phi_{j}$. Then transformations $\Phi^{j}$ are defined on $D\left(0, r_{j}\right)$. By the transformation $\Phi^{j}$, system (2) becomes

$$
\left\{\begin{array}{l}
\dot{x}_{j}=\Omega y_{j}+h_{1}^{j}\left(x_{j}, y_{j}, t\right)+f_{1}^{j}\left(x_{j}, y_{j}, t\right), \\
\dot{y}_{j}=M^{j}\left(x_{j}, y_{j}\right)+h_{2}^{+}\left(x_{j}, y_{j}, t\right)+f_{2}^{j}\left(x_{j}, y_{j}, t\right),
\end{array}\right.
$$

where $M^{j}\left(x_{j}, y_{j}\right)$ is a normal term, $h^{j}=\left(h_{1}^{j}, h_{2}^{j}\right)^{\mathrm{T}}$ is higher order term, and $f^{j}=\left(f_{1}^{j}, f_{2}^{j}\right)^{\mathrm{T}}$ is a small perturbation term. Moreover, by (16), (19) and (20) we can get

$$
\begin{gathered}
\left\|M^{j+1}-M^{j}\right\| \|_{m_{j}, \Delta\left(r_{j}, \delta_{j}\right)} \leq c \varepsilon_{j}, \\
\left\|h^{j+1}-h^{j}\right\| \|_{m_{j+1}, \Delta\left(r_{j+1}, \delta_{j+1}\right)} \leq \varepsilon_{j}, \\
\left\|f^{j}\right\|_{m_{j}, \Delta\left(r_{j}, \delta_{j}\right)} \leq \varepsilon_{j} .
\end{gathered}
$$

Let $\tilde{M}^{j}=M^{j}+\hat{M}^{j}=M^{j}+\hat{M}_{0}^{j}\left(x_{j}\right)+\hat{M}_{1}^{j}\left(x_{j}\right) y_{j}$. We apply Theorem $2.2[8]$ with $f_{0}=M\left(x_{0}, y_{0}\right)$ and $\left\{\hat{f}_{j}\right\}$, where $\hat{f}_{j}=\hat{M}_{0}^{j}$, and have $\left\{x_{(j)}\right\}$ and $\left\{f_{j}\right\}$ with $\left|x_{(j)}\right| \leq c \varepsilon_{j}^{\frac{4}{3}}$. Then we have $M^{j+1}\left(x_{j+1}, y_{j+1}\right)=\tilde{M}^{j}\left(x_{j}+x_{(j)}, y_{j}\right)$, $M_{0}^{j+1}\left(x_{j+1}\right)=f_{j+1}\left(x_{j+1}\right)$.

The domain $D\left(0, r_{j}\right)$ converges to the domain $D\left(0, \frac{r}{2}\right)$ with $\delta_{j} \rightarrow \frac{\delta}{2}$, $m_{j} \rightarrow \frac{m_{0}}{2}$. Our work is to verify that all the concerned sequence can be convergent under the norm $\|\cdot\| \cdot \|_{\frac{m_{0}}{2}}, \frac{r}{2}, \frac{\delta}{2}$.

$$
\begin{gathered}
\left\|D \Phi^{j}\right\|\left\|_{m_{j}, \Delta\left(r_{j}, \delta_{j}\right)} \leq\right\| D \Phi_{0}\|\|_{m_{j}, \Delta\left(r_{j}, \delta_{j}\right)} \cdots\left\|D \Phi_{j-1}\right\| \|_{m_{j}, \Delta\left(r_{j}, \delta_{j}\right)} \leq \prod_{i=0}^{j-1}\left(1+c \varepsilon_{i}\right) \leq c . \\
\|\| \Phi^{j+1}-\Phi^{j}\|\|_{m_{j}, \Delta\left(r_{j}, \delta_{j}\right)} \leq\left\|D \Phi^{j}\right\|\left\|_{m_{j}, \Delta\left(r_{j}, \delta_{j}\right)}\right\| \Phi_{j}-I \|_{m_{j}, \Delta\left(r_{j}, \delta_{j}\right)} \leq c \varepsilon_{j} .
\end{gathered}
$$

Thus, $\Phi^{j}$ is convergent under the norm $\|\cdot\| \|_{\frac{m_{0}}{2}}, \frac{r}{2}, \frac{\delta}{2}$. Let $\Phi^{j} \rightarrow \Phi^{*}, j \rightarrow \infty$. Then we can get

$$
\left\|\mid \Phi^{*}-i d\right\|_{\frac{m_{0}}{2}, \Delta\left(\frac{r}{2}, \frac{\delta}{2}\right)} \leq c \varepsilon_{0} .
$$

Using (22), it follows that $M^{j}$ converges to $M^{*}$ with

$$
\left\|M^{*}-M\right\| \leq c \varepsilon \text {. }
$$

From (24), we can get that

$$
\left\|f^{j}\right\|_{\frac{m_{0}}{2}, \frac{r}{2}, \frac{\delta}{2}} \rightarrow 0
$$


By (23), $\left\{h_{j}\right\}$ is convergent on $D\left(0, \frac{r}{2}\right)$ with $j \rightarrow \infty$, and

$$
h_{j} \rightarrow h^{*}
$$

under the norm $\|\cdot\| \frac{m_{0}}{2}, \frac{r}{2}, \frac{\delta}{2} \cdot$

Then, by the transformation $\Phi^{*}(\cdot, \cdot, t)$, system (2) changes to the following system

$$
\left\{\begin{array}{l}
\dot{x}=\Omega y+h_{1}^{*}(x, y, t) \\
\dot{y}=M^{*}(x, y)+h_{2}^{*}(x, y, t)
\end{array}\right.
$$

$M^{*}(x, y), h^{*}(x, y, t)$ has the same form as $M, h$.

Thus, Theorem 2.5 is proved.

\section{Acknowledgements}

We would like to thank the reviewers for their thoughtful comments and efforts toward improving our manuscript.

This research was supported by the National Natural Science Foundation of China (12171420) and the Doctoral Foundation of Zaozhuang University (1020704).

\section{Conflicts of Interest}

The authors declare no conflicts of interest regarding the publication of this paper.

\section{References}

[1] Jorba, A. and Simó, C. (1992) On the Reducibility of Linear Differential Equations with Quasiperiodic Coefficients. Journal of Differential Equations, 98, 111-124. https://doi.org/10.1016/0022-0396(92)90107-X

[2] Jorba, A. and Simó, C. (1996) On Quasi-Periodic Perturbations of Elliptic Equilibrium Points. SIAM Journal on Mathematical Analysis, 27, 1704-1737.

https://doi.org/10.1137/S0036141094276913

[3] Geng, J.S. and Wu, J. (2017) Real Analytic Quasi-Periodic Solutions with More Diophantine Frequencies for Perturbed KdV Equations. Journal of Dynamics and Differential Equations, 29, 1103-1130. https://doi.org/10.1007/s10884-016-9529-3

[4] Xu, J. and Zheng, Q. (1998) On the Reducibility of Linear Differential Equations with Quasiperiodic Coefficients Which Are Degenerate. Proceedings of the American Mathematical Society, 126, 1445-1451. https://doi.org/10.1090/S0002-9939-98-04523-7

[5] Pöschel, J. (1990) Small Divisors with Spatial Structure in Infinite-Dimensional Hamiltonian Systems. Communications in Mathematical Physics, 127, 351-393. https://doi.org/10.1007/BF02096763

[6] Xu, J.X. (2011) On Small Perturbation of Two-Dimensional Quasi-Periodic Systems with Hyperbolic-Type Degenerate Equilibrium Point. Journal of Differential Equations, 250, 551-571. https://doi.org/10.1016/j.jde.2010.09.030

[7] Kohno, T. (2017) Preconditioned Iterative Method for Regular Splitting. Advances in Pure Mathematics, 7, 180-187. https://doi.org/10.4236/apm.2017.72009 
[8] Liu, X., Du, W., Yu, Y. and Qin, Y. (2018) Efficient Iterative Method for Solving the General Restricted Linear Equation. Journal of Applied Mathematics and Physics, 6 , 418-428. https://doi.org/10.4236/jamp.2018.62039

[9] Qiu, W.H. and Si, J.G. (2013) Reducibility for a Class of Almost-Periodic Differential Equations with Degenerate Equilibrium Point under Small Almost-Periodic Perturbations. Abstract and Applied Analysis, 12, 1-9. https://doi.org/10.1155/2013/386812

[10] Afzal, M., Guo, S.Z. and Piao, D.X. (2019) On the Reducibility of a Class of Linear Almost Periodic Hamiltonian Systems. Qualitative Theory of Dynamical Systems, 18, 723-738. https://doi.org/10.1007/s12346-018-0309-9 